\title{
BILATERAL COORDINATION ON PHYSICAL EDUCATION ACTIVITIES AND GROSS MOTOR SKILLS AT AN INTERNATIONAL SCHOOL IN KRABI
}

\author{
Tiraka Nualjan \\ A-Chuan Kindergarten School, Krabi, Thailand \\ fenwickinterschool@hotmail.com
}

\begin{abstract}
:
Children develop gross motor skills at different rates, but when young children have difficulty with these skills, they can perform different primary motor activities. This has an effect on further learning. The school helps develop English learning activities for students in kindergarten Krabi Province, Thailand. By applying bilateral coordination practice with learning the basic English language knowledge in Physical Education Activities. We found that students were more interested and able to develop learning skills in all aspects of the age with fun and can increase learning English and basic academic effectively. And also found that the physical attributes of balance and bilateral coordination also help to provide appropriate posture for tabletop tasks and to keep kids focused, it also has an effect on the success of the later times in small motors. We conclude by encouraging theoretical and methodological approaches to clarify the mechanisms that implicate bilateral coordination on Physical Education Activities and Gross Motor Skills in school performance and achievement.
\end{abstract}

Key words: - Bilateral coordination, Physical Education Activities, Gross Motor Skills.

\section{INTRODUCTION:}

\section{Introduction}

Having a wide range of skills makes it possible to learn more about the world around them and increase their knowledge effectively. Learning gross motor skills is an important starting point for preschoolers and is very helpful in further development of skills for the purpose of this education is to develop cognitive skills in kindergarten. By applying the relationship between Primary motor skills and advanced motor skills in relation to bilateral coordination in Physical Education Activities. At the Early stage of childhood, it's important to note that this marks the period for coordinating a child bilaterally. This will enhance the rapid adaptation in relation to sport and motor skills. Through sport and motor skills children would have the ability to use both side of their body in a coordinated way. Bilateral coordination refers to the ability to coordinate both sides of the body at the same time in a controlled and organized manner, for example; stabilizing paper with one hand while writing/cutting with the other. It is the ability to use both sides of body in an integrated and skillful manner [1]. The Bilateral Coordination subtest measures the motor skills involved in playing sports and many recreational games. The tasks require body control, and sequential and simultaneous coordination of the upper and lower limbs. Sample's score is consistent with individuals who can perform coordinated arm/hand and leg/foot movements when the limbs on the same sides of the body are synchronized, but have difficulty with coordinated arm/hand and leg/foot movements when the limbs on the opposite sides of the body are synchronized [2]. Good bilateral coordination/integration is an indicator that both sides of the brain are 
communicating effectively and sharing information [3]. Early childhood curriculum and pedagogical approaches aim to promote holistic attitudes to teaching and learning which recognize the important contribution a child's physical, cognitive, social, and emotional development has on their learning and readiness to start school [4]. For example, a recently published systematic review by Donnelly et al. [5] comprehensively summarized the findings of studies examining the relationships between PA, fitness, cognitive function, and academic achievement. The synthesis included a summary of findings from 27 observational studies that examined the relationship between physical fitness and academic achievement in children aged five to 13 years, demonstrating largely positive findings, although it highlighted several limitations in relation to study quality and reporting that resulted in inconsistent findings. Notably, the components of physical fitness measured by these studies included CRF, muscular strength and endurance, flexibility, and body composition. However, as engagement in PA is dependent not only on health-related physical fitness but also on performance-related physical fitness, which we have termed 'motor proficiency' in this review, further examination of the relationships between motor proficiency, cognition, and academic performance is warranted, as reviews to date on these relationships have been scant [6]. Significant positive associations were also evident between academic performance and components of gross motor proficiency, specifically speed and agility, upper-limb coordination, and total gross motor scores. Preliminary evidence from a small number of experimental studies suggests motor skill interventions in primary school settings may have a positive impact on academic performance in mathematics and/or reading [7]. We focus on the development of kindergarten children. In physical education by organizing activities bringing bilateral coordination training and Gross Motor Skills. Applied with other learning subjects such as color classification. It is an activity management with multiple steps in one activity linked together an International School in Krabi

\section{MATERIAL \& METHODS:}

Bilateral coordination requires movements of both sides of the body, especially movements of both hands together in activities require processing and integration of both hemispheres of the brain to enable both hands working together at the same time. Balance is extremely important in different sports, and it is defined as the ability of taking and keeping the balance position in static or dynamic mode. Coordinated bilateral movements engage different sensory and motor areas on both hemispheres of the brain as well as the corpus callosum and cerebellum. Those parts of the brain work together to help to produce the complex bilateral movements that make up so much of our daily lives. When bilateral integration is poor, then the child may avoid these activities, or the child may seem clumsy, inefficient and uncoordinated when taking part in them. Below are just a few of the many activities that need both sides of the body to work together well.

Kid needs to develop bilateral coordination skills in three different areas: symmetrical movements, reciprocal movements, and movements requiring a supporting hand. Symmetrical bilateral movements are usually the first ones to be mastered, followed by reciprocal movements and lastly the asymmetrical movements of a leading and supporting hand. However, your child does not 
need to completely master one kind of movement before moving on to the next - there is a lot of overlap and interdependence in the development of coordination skills. It is important that both sides of the body do the same movement at the same time, with an equal amount of force. Reciprocal movements are actions where first one hand or leg and then the other carries out the same movement in a rhythmical way. Children need a range of skills to transition successfully to formal schooling. In early childhood classrooms, children must master their fine and gross motor skills. The ability to coordinate both sides of the body together is an important skill for many motor skills. Gross motor skills such as walking, climbing stairs, running, skipping, hopscotch and jump rope all require bilateral coordination skills. Fine motor skills such as holding paper while cutting with scissors or lacing beads require the right and left sides of the body to work together. Many functional skills require both hands and both legs to work together such as buttoning, zipping, using a fork and knife, using a rolling pin and more. In addition, bilateral coordination helps to develop hand dominance (whether you are right or left-handed). The method was hypothetic-deductive was tested: Students' Bilateral Coordination will improve learning skills in all aspects of the age with fun and can increase learning English and basic academic effectively. We organize different activities every day for 3 months. The trial used 60 students. Examples of relationships of skills in one activity shown with the pictures below.

\section{RESULT \& DISCUSSION:}

Bilateral coordination activities are essential for coordinated and fluid movements that require both sides of the body. This activity involved about three to four deferent steps, run, jump, colors and balance. It will help the children to be competitive and focused on track when moving their body or trying to jump in the bags. Bilateral coordination and balance have shown that sports is very important. Jumping in the bags help them to physically lift up their body while on motion and run to pick up the balls to place them on the corns. Kids can learn about colors as they will be instructed by the teacher to place the balls on the corns in other to match the colors as numbered on the corns. Balance and coordination are two of the most important gross motor skills in a child's physical development. It allows children to participate in sports and physical activities, it reduces their risk of injury and improves their ability to perform everyday tasks. Intervals runs are like hit workouts: kids work at high intensity for a short period of time, recover, and do it again. Another important foundation in the development of bilateral coordination is body awareness. Body awareness refers to the ability to know where your body is in space without necessarily using vision i.e., how high to lift your leg when jumping, running, climbing stairs, etc. some of these activities' kids do to develop physically, mentally and health wise. It is also known as bilateral integration. Many childhood and school activities require your child to be able to use both hands together well. A child who is delayed in developing bilateral coordination skills may prefer to use one hand alone rather than both hands together, and may appear awkward or clumsy in some gross and fine motor activities. Studies have also found that there is a link between bilateral motor skills and academic performance. Coordination exercises may stimulate the parts of the brain that are needed for thinking and for paying attention. During the third month of the experiment, the children were able to 
understand for themselves what to do, what to do first, after walking, move more quickly, move more quickly. Observe from the daily life. Interesting results were found, students have fun and are able to memorize words and become more focused. When we observe behavior from learning in the classroom by doing more than 1 step of the activity, children can understand it quickly.

\section{CONCLUSION:}

The results confirmed the hypothesis that students's bilateral coordination will improve learning skills in all aspects of the age with fun and can increase learning English and basic academic effectively. Several studies have investigated the brain substrates of motor imagery. The motor ideation is controlled by a cortical network mainly involved in abstract thinking, cognitive and motor control, semantic and visual imagery processes [8]. A health-related physical education curriculum can provide students with substantially more physical activity during physical education classes. Improved physical education classes can potentially benefit $97 \%$ of elementary school students [8]. With this research Therefore can be used as part of the reason That makes the development of these skills related to academic learning skills.

\section{REFERENCES:}

T. Balakrishnan, Chavan Sasidhar Rao. Interrater Reliability of Bilateral Coordination of Bruininks

Oseretsky Test of Motor Proficiency (BOTMP) \& Performance of Indian Children Compared With USA Norms. The Indian Journal of Occupational Therapy 2007;XXXVIII(3)

Robert H. Bruininks, PhD, \& Brett D. Bruininks, PhD. Bruininks-Oseretsky Test of Motor
Proficiency, Second Edition, Complete Form Report 2013.

Magalhaes LC, Koomar JA, Cermak SA, Bilateral motor coordination in 5- to 9year-old children: a

pilot study. American Journal of Occupational Therapy

1989;43(7): 437-43.

Australian Government Department of Education, Employment and Workplace. Belonging, Being

and Becoming: The Early Years Learning Framework for Australia; Commonwealth of Australia: Canberra, VIC, Australia, 2009.

Donnelly, J.E.; Hillman, C.H.; Castelli, D.; Etnier, J.L.; Lee, S.; Tomporowski, P.; Lambourne, K.;

Szabo-Reed, A.N. Physical activity, fitness, cognitive function, and academic achievement in children: A systematic review. Med. Sci. Sports Exerc. 2016, 48, 1197-1222

Haapala, E.A. Cardiorespiratory fitness and motor skills in relation to cognition and academic

performance in children-A review. J. Hum. Kinet. 2013, 36, 55-68.

Kirstin Macdonald ,Nikki Milne ,Robin Orr and Rodney Pope Relationships between Motor Proficiency

and Academic Performance in Mathematics and Reading in School-Aged Children and Adolescents: A Systematic Review. Int. J. Environ. Res. Public Health 2018, 15(8), 1603

Simona Gardini, Annalena Venneri, William Jonathan McGeown, Cristian Toraci, Luca Nocetti, Carlo

Adolfo Porro \& Paolo Caffarra Brain Activation Patterns Characterizing Different Phases of Motor Action: Execution, Choice and 
Ideation. Brain Topography volume 29, pages679-692(2016)

J F Sallis, T L McKenzie, J E Alcaraz, B Kolody, N Faucette, and M F Hovell The effects of a 2-year physical education program (SPARK) on physical activity and fitness in elementary school students. Sports, Play and Active Recreation for Kids. Published Online: October 07, 2011
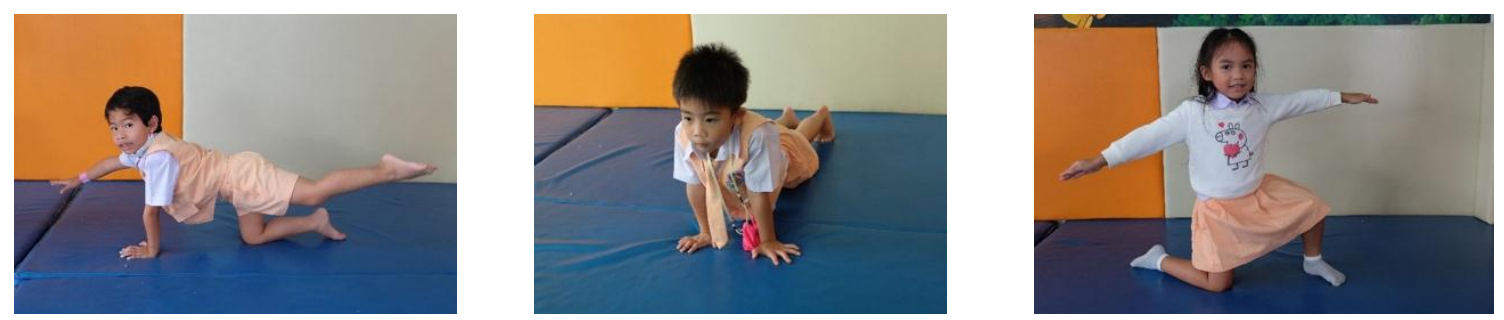

Figure 1: Students performing reciprocal movements
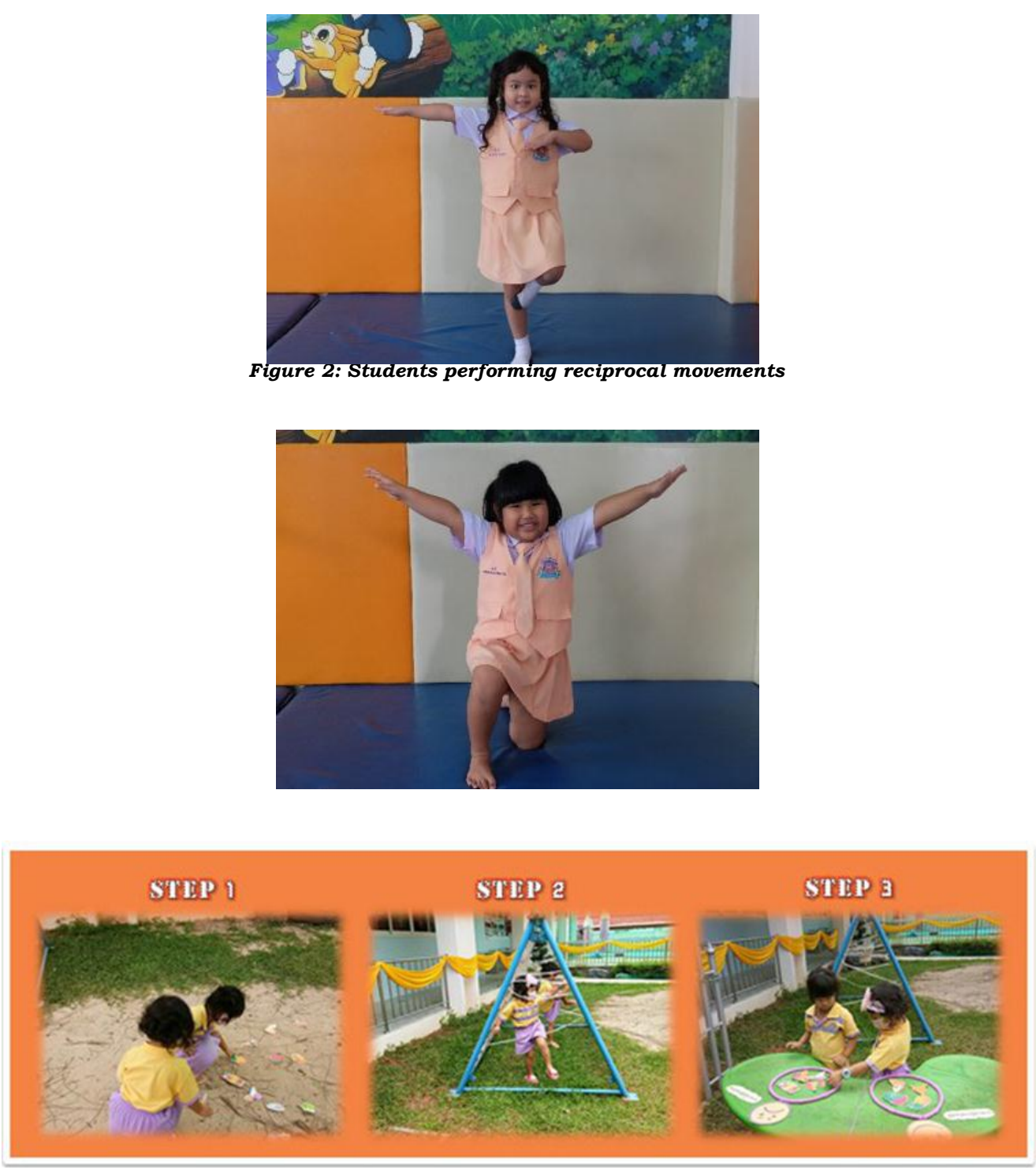
Figure 4: Students performing symmetrical movements with the topics they learnt

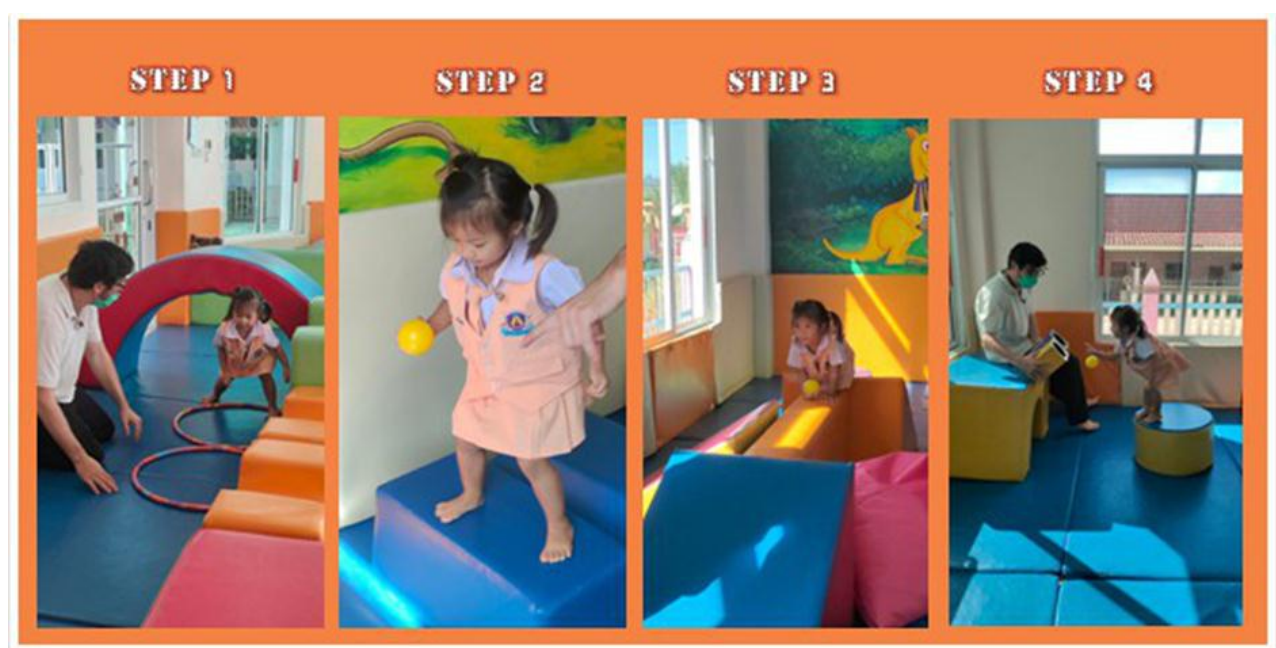

Figure 5: Student's body is lifted up as she jumps, lifts up the ball and places it in the corns.

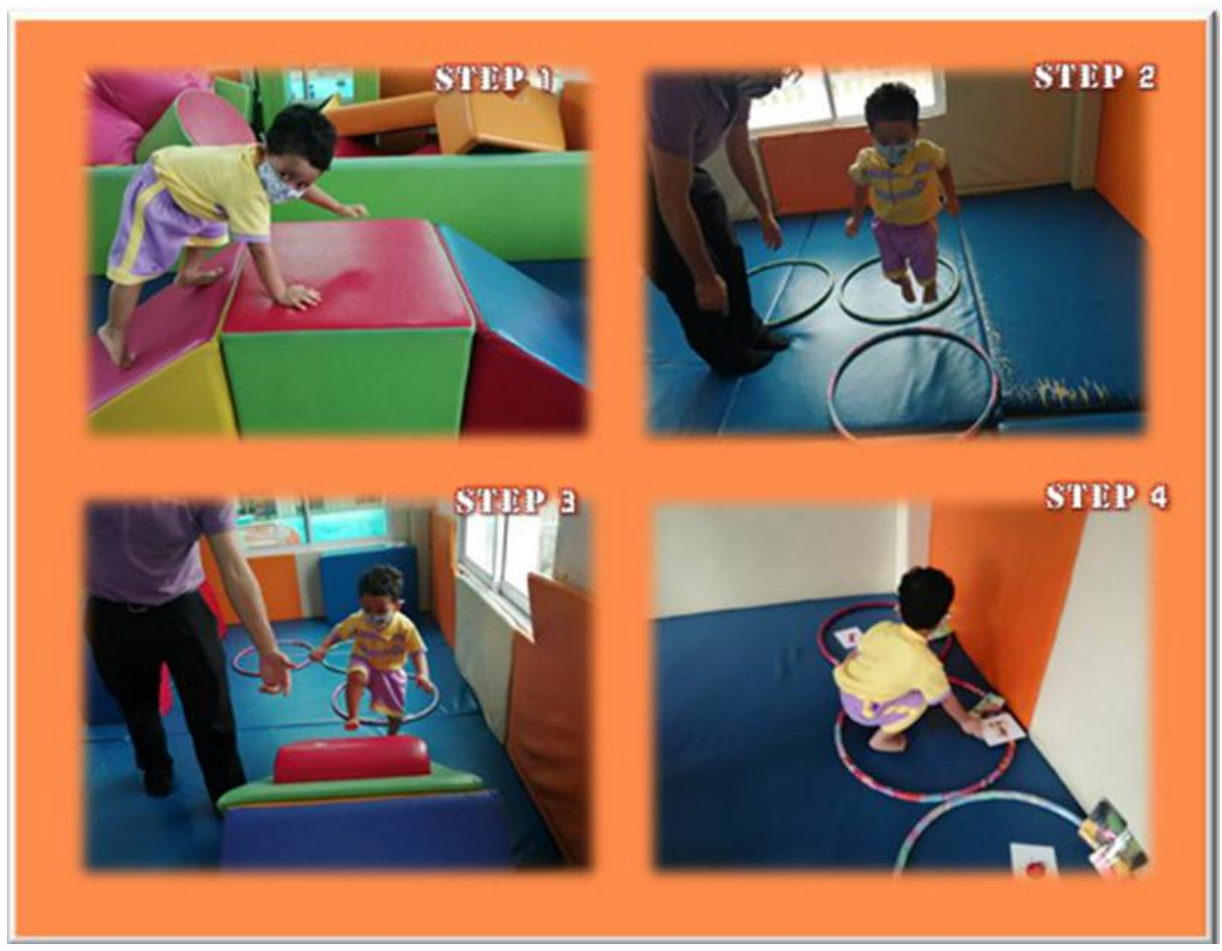

Figure 6: Students engaging in balance and coordination activities like balancing his body on a block, jumping in a circle, running upwards on a block and matching the correct cards 


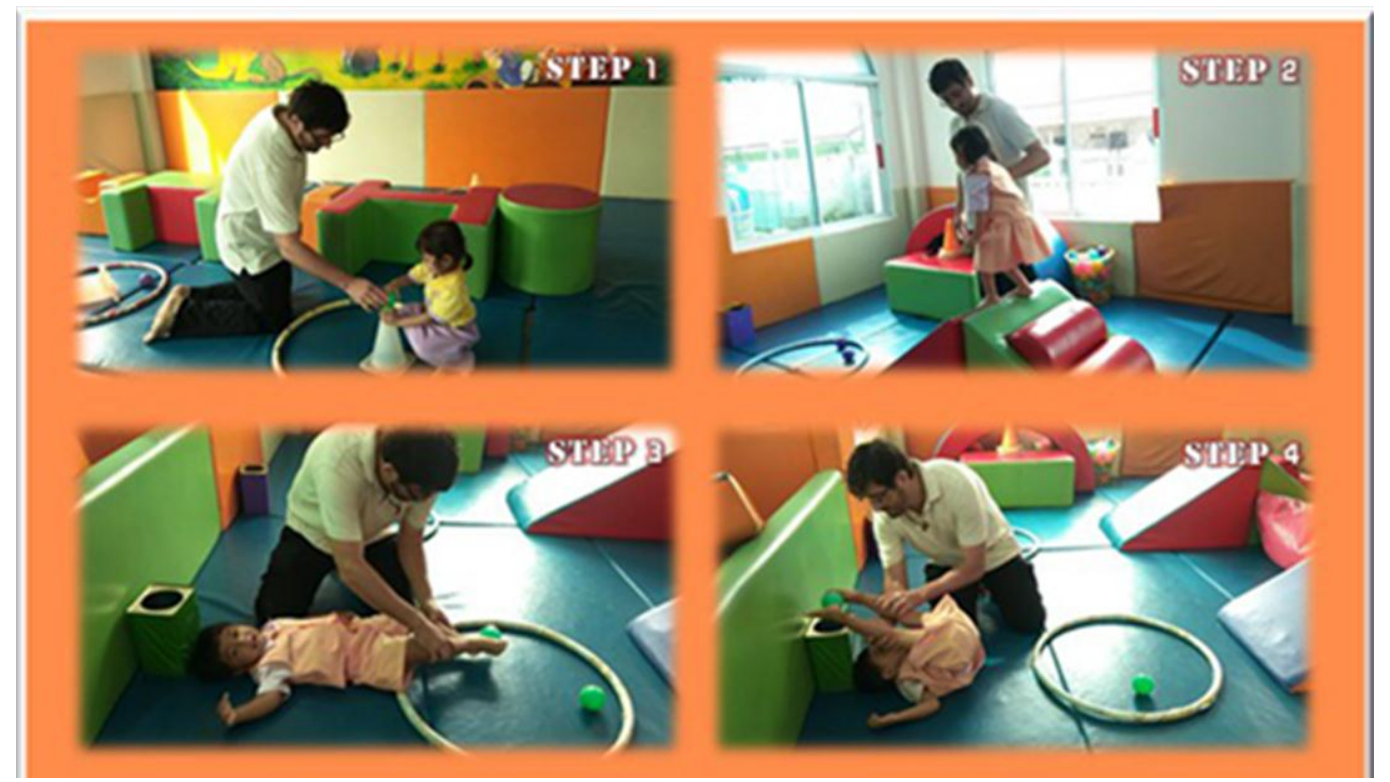

Figure 7: Students acquire body awareness when they perform activities like placing a ball, climbing the stairs, clutching and moving a ball with their legs

FINDINGS:

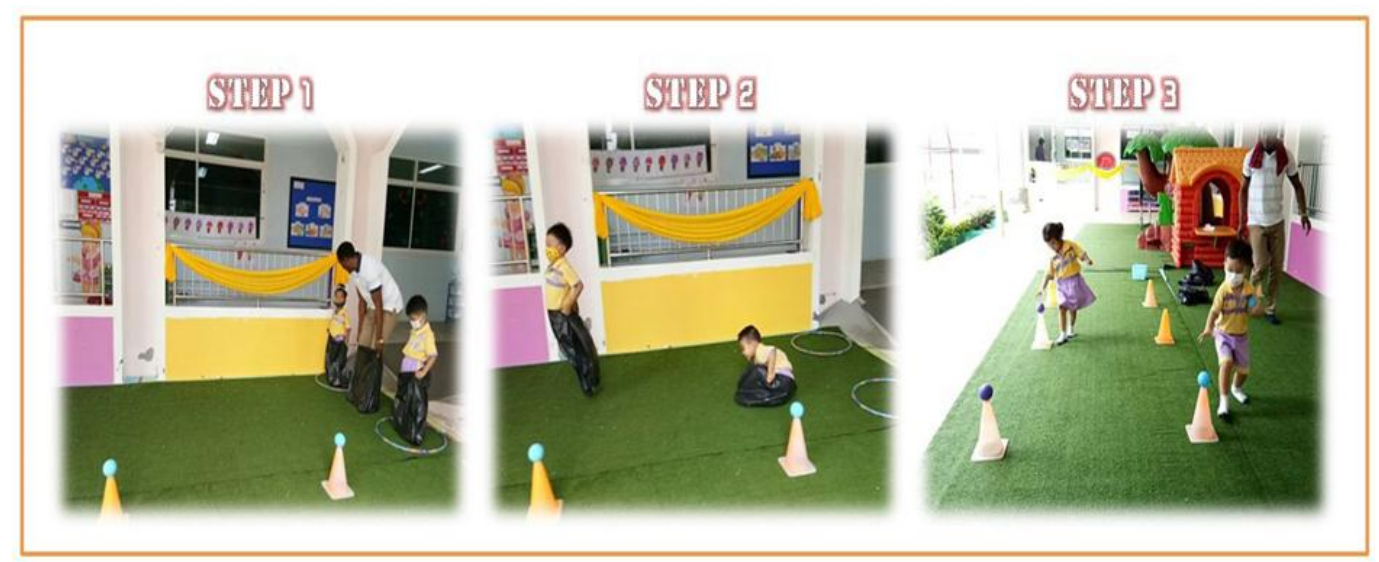

Figure 8: Students performing bilateral coordination activities like jumping in the bag and running to pick up the balls 